\title{
Introduction to the Handbook of Research Methods and Applications in Experimental Economics
}

Arthur Schram and Aljaž Ule

Economics is an experimental science. Most modern universities now host a laboratory for economic experiments and many scholars who use the laboratory are also involved in field experiments. In addition, the field of neuroeconomics, a discipline that often uses laboratory experiments, is starting to gain ground in many universities.

Though the field is relatively young, the use of laboratory and field experiments has grown exponentially since the pioneers in experimental economics started the discipline five decades ago. The organization of experimentalists, the Economic Science Association (ESA), was founded in 1986. While the first ESA Meetings in Tucson, Arizona witnessed fewer than 50 papers (Svorenčík, 2015), the 2018 International ESA Meetings in Berlin had a program with over 550 participants. The ESA started its own journal (Experimental Economics) in 1998, followed by a second journal (the Journal of the Economic Science Association) in 2015. Together these journals published 56 papers in 2017. This comes on top of the many experimental papers published in other journals (including 133 in the top five journals in economics in the five years between 2013 and 2017; see Gërxhani and Gereke, 2019).

In short, experimental economics has become a large field. It is also a field where methodological discussions were rare, for a long time. From the beginning, there has been strong agreement about important methodological issues, such as the use of incentives and the ban on deception (see, for example, one of the first text books, Davis and Holt, 1993). Only over the past decade or two have methodological discussions about the experimental method become more common (e.g., a special issue of the Journal of Economic Methodology in 2005). However, they remained sporadic and partial and the discipline lacked a systematic overview of its methodological challenges and tools. This volume fills this gap.

For this Handbook, we have gathered a variety of new methodological studies and discussions relevant for experimental economics in a broad sense. This includes laboratory and field experiments and methods related to neuroeconomics. Each chapter is an original contribution. We have aimed at a mix of established, leading authorities and young, talented scholars. Our goal was to combine practical methodological overviews, such as on software and recruitment tools, with discussions of important choices like deception and incentives, and relatively new developments such as replication and the emergence of novel equilibrium concepts. This has led to a division of this volume into five parts, devoted respectively to the basic methods of experimental economics, field experiments, neuroeconomics, applications to economic theory and policy, and experimental procedures.

Throughout this volume, methodological issues at the core of experimental design and procedures are discussed. To set the stage for what follows, we list here a brief overview of various strengths and weaknesses of the experimental method in economics. Most 
of the perceived strengths of experimentation are directly related to the control that the researcher has over the environment in which participants make decisions. Inter alia, control allows for:

- induced preferences, that is, "using a reward structure to induce prescribed monetary value on actions" (Smith, 1976, p. 275), which provides control over participants' incentives (see Chapter 1 of this volume);

- ceteris paribus testing of theories, that is, changing a single factor at a time between experimental treatments to test the theoretically predicted effects (Schram, 2005);

- testing causal effects, that is, directly measuring the effect on an outcome of changing a single variable;

- measuring data that are unavailable in the field, such as beliefs (see Chapter 3);

- true randomization when testing a theory that assumes random events (see Chapter 7);

- replicating previous results by rerunning the same experiment (see Chapter 5);

- wind-tunnel testing, that is, testing policy proposals before their large-scale implementation (see Chapter 15).

Most of the weaknesses attributed to experiments are related to a low external validity, that is, the idea that the experimental environment insufficiently reflects the situations that prompted the research. In this respect, field experiments are perceived to have higher external validity than laboratory experiments (see Falk and Heckman, 2009 and Camerer, 2015 for opposing points of view, however). Inter alia, diminished external validity could arise from the following:

- The typical experiment has low numbers of observations, though field experiments tend to have more than laboratory experiments. Of course, this is not a weakness of the method per se, but more of the implementation of the method. Proper statistical testing can help to circumvent this problem (see Chapters 4 and 10). The consequences of low power for experiments in macroeconomics are discussed in Chapter 14.

- Subjects might behave differently when they are aware of taking part in an experiment. This problem can often be circumvented in field experiments by hiding the experiment (Chapters 6 and 7). It might be aggravated if there are experimenter demand effects, but these seem rare in experimental economics (as argued in Chapter 20).

- Experimental behavior might differ from one subject pool to another. This is especially important in subject pools from distinct cultures (see Chapter 18).

We now provide an overview of the parts and chapters of this volume.

\section{PART I: METHODS OF EXPERIMENTAL ECONOMICS}

Chapters 1-5 discuss the basic methods of experimental economics. There has long been wide agreement about the basic principles for experimentation in economics, such as the use of incentives and a ban on deception. In this part, contributions discuss and elaborate on such traditional views and present recent developments. 
In Chapter 1, James Cox and Vjollca Sadiraj discuss incentives, one of the key concepts of economics in general and experimental economics in particular. Most economic experiments use monetary incentives that are salient and vary with participants' actions. This intends to engage participants in ways that reflect how they engage in relevant external economic decisions. Incentives are traditionally seen as a crucial part of experimental control. This chapter outlines six complications that might confound control. It discusses their interaction with experimental methods and approaches to control for confounds.

In Chapter 2, Andreas Ortmann addresses the issue of deception. This is one of the most disputed topics in experimental social sciences. In contrast to social psychology, economists have essentially banned the use of deception in the laboratory, though disagreement remains about what constitutes deception. This chapter reviews various forms of deception, their pros and cons, and the experimental evidence on their effects. It also explores the extent to which traditional objections to the use of deception in laboratory experiments carry over to field experiments.

Chapter 3 by Hande Erkut and Ernesto Reuben discusses preference measurement and manipulation. In economic theory, individual choices are commonly assumed to be driven by preferences that are rationalizable by a utility function. The predictive ability of most microeconomic models relies on properly quantifying these preferences and on their rationalizability. This chapter reviews methodologies used in experimental economics to measure preferences and their degree of consistency with utility maximization. Topics include the measurement of risk, intertemporal, and social preferences.

In Chapter 4 on the analysis of experimental data, Peter Moffatt describes and illustrates a number of techniques used in the econometric analysis of data from economic experiments. To illustrate the techniques, the chapter uses experiments on choice under risk. The techniques range from the direct treatment testing, through intermediate techniques such as probit estimation, finding predicted probabilities and marginal effects, and interval regression, to a fairly advanced set of techniques that are applicable when repeated decisions are available from each subject. The pros and cons of various methods of data analysis are discussed.

Chapter 5 by Colin Camerer, Anna Dreber and Magnus Johannesson is the first overview of replication studies and related techniques in experimental economics. The recent "replication crisis" in the social sciences has highlighted the need for high-powered replications to confirm original results before strong conclusions can be drawn. This chapter reviews two big replication projects in psychology and economics and assesses their results in terms of the various replication indicators. It further illustrates how prediction markets and surveys have been used to predict replication outcomes. The chapter ends with a discussion of general quality control in research, and opinions on improved practices.

\section{PART II: FIELD EXPERIMENTS}

Chapters 6 and 7 are on the methods in field experiments. Over the past two decades, experimental economics has seen a large increase in the use of field experiments. Field experiments can provide a useful bridge between the stylized environment of the laboratory and the context-rich environment of the outside world. Field experiments have now been used in a range of applications, including in development economics, charitable 
giving, labor economics, discrimination in markets, financial decision-making, education and health.

In Chapter 6, Anya Samek presents advantages and disadvantages of field experiments in relation to laboratory experiments. In a choice between laboratory and the field there is often a trade-off between generalizability and control. Generalizability can sometimes be higher in the field, but this comes with some loss of control. Aside from focusing on this trade-off, this chapter characterizes types of field experiments, provides a useful set of tips for the collaboration with external partners and a how-to guide for researchers new to the method.

Chapter 7 is a contribution by Noemi Peter and Adriaan Soetevent on randomization in field experiments. In the field, researchers face constraints in the design they can implement and/or the type of data that can be collected. The chapter discusses possible ways to tackle issues related to randomization, using examples from articles published in top economics journals. It addresses treatment spillovers, implementation and practical issues when selecting the level of randomization, and the use of covariates in the design and analysis stages. An insufficient control of the assignment mechanism or partial treatment compliance may lead to failures and a discussion is included of how this can be prevented.

\section{PART III: NEUROECONOMICS}

Chapters 8-11 are dedicated to neuroeconomics. Economic decisions are fundamentally linked to our biology and it is therefore essential to investigate the biological mechanisms underlying economic decision-making. Neuroeconomics explores the neural basis of economic models, using physiological data such as brain images, hormones, and DNA. Its goal is to provide mechanistic explanations about how neural functions lead to cognitive and emotional processes behind economic decisions.

Chapter 8 by Jan Engelmann, Manon Mulckhuyse and Chih-Chung Ting introduces the most important brain measurement and manipulation methods that are commonly used in neuroeconomics. The chapter highlights recent experiments to illustrate applications of these methods and their specific advantages and limitations. It provides a brief overview of neuronal communication, which can be captured as a looking glass into the brain; "correlational" methods like functional magnetic resonance imaging (fMRI) that measure neuronal activity during behavioral tasks; and a manipulation that allows disruption of specific brain regions so that their contribution to a behavior of interest can be identified.

In Chapter 9, Oliver Hulme, Edward Webb and Alexander Sebald introduce physiological economics, focusing on hormonal signals that can be sampled from blood. The chapter starts with a basic primer on endocrinology. This includes a description of the commonly analyzed hormones, and a discussion of their biological and economic relevance and measurement. It then describes the evidence on the relation between hormones and economic behavior, finally exploring limitations, challenges, and potential future directions for the area.

Chapter 10 on data analysis in neuroeconomics is by Mael Lebreton and Kerstin Preuschoff. It introduces the analysis of the most frequent data types such as those from functional magnetic resonance imaging (fMRI), magnetoencephalography (MEG) or 
electroencephalography (EEG). Aimed at non-specialists in this field, the analysis is illustrated by example studies investigating the representation of utility in the human brain. The chapter provides practical advice for interpreting neuroimaging results, and includes links to key references and dominant analysis packages used in the neuroeconomics community.

In Chapter 11, Carsten De Dreu and Jörg Gross pursue the integration of personality psychology with economic preferences and choice. They first discuss when and how behavior can be identified as emanating from stable personality differences. They review the psychometric methods used in personality psychology and a selective set of personality variables captured in the Big Five theory of personality and the biobehavioral approach/ avoidance system. Then, they examine how the methodology of personality psychology can be applied in the realm of economic preferences and their measurement.

\section{PART IV: DEVELOPING ECONOMIC THEORY AND POLICY}

Chapters 12-15 illustrate how experimental methodology is applied to developing economic theory and policy. Experiments have yielded important insights into the systematic deviations from strict rationality and into the heterogeneity of preferences among decision-makers that can form the basis for the development of theory as well as for the design of policies.

In Chapter 12, Graham Loomes argues that it is important to take into account the process by which individuals arrive at their decisions when modeling risky choice. Most theories of risky choice model individuals as if they have precise and stable preferences so that their decisions will be made relatively effortlessly and will exhibit high degrees of consistency and reliability. Loomes argues that the experimental evidence suggests differently, however. For most people there will be numerous cases where they are uncertain about their preferences, where their decisions may change from one occasion to another, where they may find it difficult to decide, or where, having decided, they are less than fully confident that they made the right choice. This chapter discusses how processes behind decisions might be modeled and considers some of the implications for future directions in the development of decision theories.

Chapter 13 by Jacob Goeree, Charles Holt, Philippos Louis, Thomas Palfrey and Brian Rogers proposes a non-parametric generalization of the quantal response equilibrium (QRE), accounting for errors in an equilibrium analysis of games. One objection to QRE is that specific functional forms must be chosen to derive equilibrium predictions. As these can be chosen from an infinitely dimensional set, another concern is whether QRE is falsifiable. Finally, QRE can typically only be solved numerically. This chapter addresses these concerns through a novel set-valued solution concept, rank-dependent choice equilibrium (RDCE). Important differences between RDCE and QRE are discussed, showing that RDCE envelopes all QRE models and that both are falsifiable.

In Chapter 14, Camille Cornand and Frank Heinemann discuss the methods used in laboratory experiments that can be useful for macroeconomics. They identify a number of advantages, such as policy testing and accounting for strategic uncertainty, biases and heterogeneity, and then explain how dynamic stochastic general equilibrium (DSGE) models are implemented in laboratory experiments and how heuristic switching models 


\section{Handbook of research methods and applications in experimental economics}

can be used to analyze out of equilibrium expectations. They also review the tests of the microfoundations of DSGE models and the methods of testing consumption smoothing and informational frictions, finally discussing the challenges and limitations for experimental macroeconomics.

Chapter 15 by Peter Werner and Arno Riedl argues for the value of experimental evidence in policy design, illustrated by applications to environmental and tax policy. Experiments can serve as "testbeds" to compare alternative market rules and mechanisms under tightly controlled conditions, which may substantially improve ex ante predictions about the outcomes of policy interventions. Experiments also lead to improved theories of decision-making behind the policy design. The chapter reviews the evidence and gives directions for successful collaborations between experimental economists and political decision-makers.

\section{PART V: EXPERIMENTAL PROCEDURES}

Chapters 16-21 present six contributions that review and discuss laboratory procedures, from recruitment and computer software, to the design of real-effort tasks and communication tools, and the challenges of cross-cultural research or related to perceived experimenter demand.

In Chapter 16, Ben Greiner and Marianne Stephanides discuss issues related to recruitment and subject pools, such as research ethics, sample size planning, recruitment selection bias, and subject pool management. They start by exploring the history and application of ethical principles regarding human-subjects research to economic experiments. Then, they discuss the tools for determination of necessary sample sizes in order to balance power, error probabilities, and experimenter budget, taking a short recourse to the basics of null hypothesis testing. They also consider evidence for recruitment selection bias, at which stages it may be effective, and how to possibly overcome it. Subsequently, they discuss recruitment issues for different types of human subjects, and tools to manage these subject pools. They conclude with practical recommendations for ethically correct, selection-bias minimizing, and efficient recruitment procedures.

In Chapter 17, Joep Sonnemans and Ailko van der Veen discuss the organization of an experimental economics laboratory, and the use of experimental software tools. They distinguish between "software tools" and experimental "programs." Software tools such as z-Tree, oTree, PHP and JavaScript offer a programming environment, while the programs define the logic of a specific experiment. The chapter starts with a discussion of laboratory organization, emphasizing the need for clear rules, a logbook, and a central repository for programs and protocols so that they can be reused. Then they review selection criteria for software tools, such as adaptability and scalability. They finally apply these criteria to three available software tools.

Chapter 18, on cross-cultural experiments, is by Christian Thöni. Conducting identical experiments with subjects from different cultures allows a robustness test of assumptions and conclusions in behavioral economics. Some experimental observations replicate across cultures, but others appear to be sensitive to local norms and institutions. Crosscultural experiments offer an investigation of social determinants of behavior under unmatched control over the environment in which behavior is observed. Cross-cultural 
experimentation also poses a number of methodological challenges, because culture cannot be randomly assigned to participants. This chapter provides a review and discussion of the methods used in cross-cultural behavioral experiments and surveys the related literature, offering a reanalysis of resulting data by investigating the importance of cultural variation relative to subject pool variation.

In Chapter 19, Jeffrey Carpenter and Emiliano Huet-Vaughn review the use of realeffort tasks in economic experiments. With a focus on principal-agent relationships, they first discuss the rationales for choosing between real and chosen effort when designing an experiment. To facilitate this discussion, they present a taxonomy of the common tasks that have been used in laboratory experiments, raise attention to issues that need to be considered when implementing them, and survey the limited literature that compares real against chosen effort tasks. They finally offer recommendations on related topics that could use additional investigation.

Chapter 20 by Jonathan de Quidt, Lise Vesterlund and Alistair Wilson considers experimenter demand effects, a phenomenon where experimental participants shift their behavior in response to their speculations about the hypothesis motivating the experiment. Such effects threaten both the internal and external validity of a study. The authors argue that this threat is taken seriously by experimental economists, who have developed a number of best practices to suppress or eliminate demand effects. The chapter outlines these best practices and reviews the literature to show that they are followed in the clear majority of published work, limiting the evidence of such effects. Finally, the chapter discusses methods to derive bounds on experimenter demand effects.

In the final chapter, Chapter 21, Jordi Brandts, David Cooper and Christina Rott discuss issues about the implementation of communication in the laboratory, such as variations in the channels and structure of communication. A particular focus is on the coding and analysis of free-form communication. A number of studies are then surveyed in which behavior is compared between free-form and more restricted communication. The chapter concludes with a brief discussion of why communication matters, and of the mechanisms through which communication affects behavior.

\section{REFERENCES}

Camerer, Colin. 2015. "The promise and success of lab-field generalizability in experimental economics: a critical reply to Levitt and List." In Guillaume R. Fréchette and Andrew Schotter (eds), Handbook of Experimental Economic Methodology (pp. 249-95). Oxford: Oxford University Press.

Davis, Douglas D. and Charles A. Holt. 1993. Experimental Economics. Princeton, NJ: Princeton University Press.

Falk, A. and James J. Heckman. 2009. "Lab experiments are a major source of knowledge in the social sciences." Science 326(5952): 535-8.

Gërxhani, Klarita and Johanna Gereke. 2019. "Experimental economics and experimental sociology.” Forthcoming in The Oxford Research Encyclopedia of Economics and Finance. New York: Oxford University Press.

Schram, Arthur. 2005. "Artificiality: the tension between internal and external validity in economic experiments." Journal of Economic Methodology 12(2): 225-37.

Smith, Vernon L. 1976. "Experimental economics: induced value theory." American Economic Review 66(2): 274-9.

Svorenčík, Andrej. 2015. "The experimental turn in economics: a history of experimental economics." Utrecht School of Economics Dissertation Series No. 29. 